Supplemental data for:

\title{
Evaluation of targeting efficiency of joints with anti- collagen II antibodies
}

Laren A. Lofchy ${ }^{1,2 \#}$, Vivian P. Vu ${ }^{1 \#}$, Nirmal K. Banda ${ }^{3,4}$, Joseline Ramos Ramirez ${ }^{3}$, Weston J. Smith ${ }^{1}$, Geoffrey Gifford ${ }^{1}$, Hanmant Gaikwad ${ }^{1}$, Robert I. Scheinman, ${ }^{4,5}$ and Dmitri Simberg

${ }^{1}$ Translational Bio-Nanosciences Laboratory, The Skaggs School of Pharmacy and Pharmaceutical Sciences, University of Colorado Anschutz Medical Campus, Aurora, CO, 80045, USA

${ }^{2}$ Graduate program in Pharmaceutical Sciences, The Skaggs School of Pharmacy and Pharmaceutical Sciences, University of Colorado Anschutz Medical Campus, Aurora, CO, 80045, USA

${ }^{3}$ Division of Rheumatology, School of Medicine, University of Colorado Denver, Anschutz Medical Campus, Aurora, CO, 80045

${ }^{4}$ Colorado Center for Nanomedicine and Nanosafety, University of Colorado Anschutz Medical Campus, Aurora, CO, 80045, USA

${ }^{5}$ Department of Pharmaceutical Sciences, The Skaggs School of Pharmacy and Pharmaceutical Sciences, University of Colorado Anschutz Medical Campus, Aurora, CO, 80045, USA

\footnotetext{
${ }^{1}$ Corresponding author

\# Equal contribution
} 


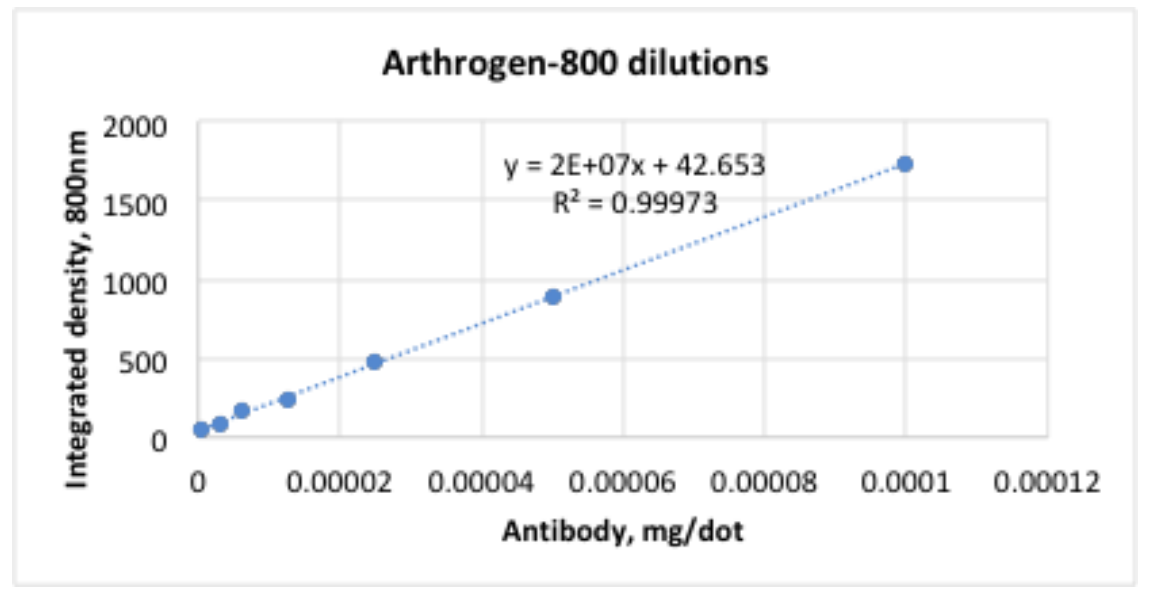

Fig. S1. Linearity of dot blot assay: Arthrogen- 800 was blotted at different concentrations on a nitrocellulose membrane and the latter was scanned with Li-COR Odyssey. The integrated density of the blot linearly increases with concentration. 


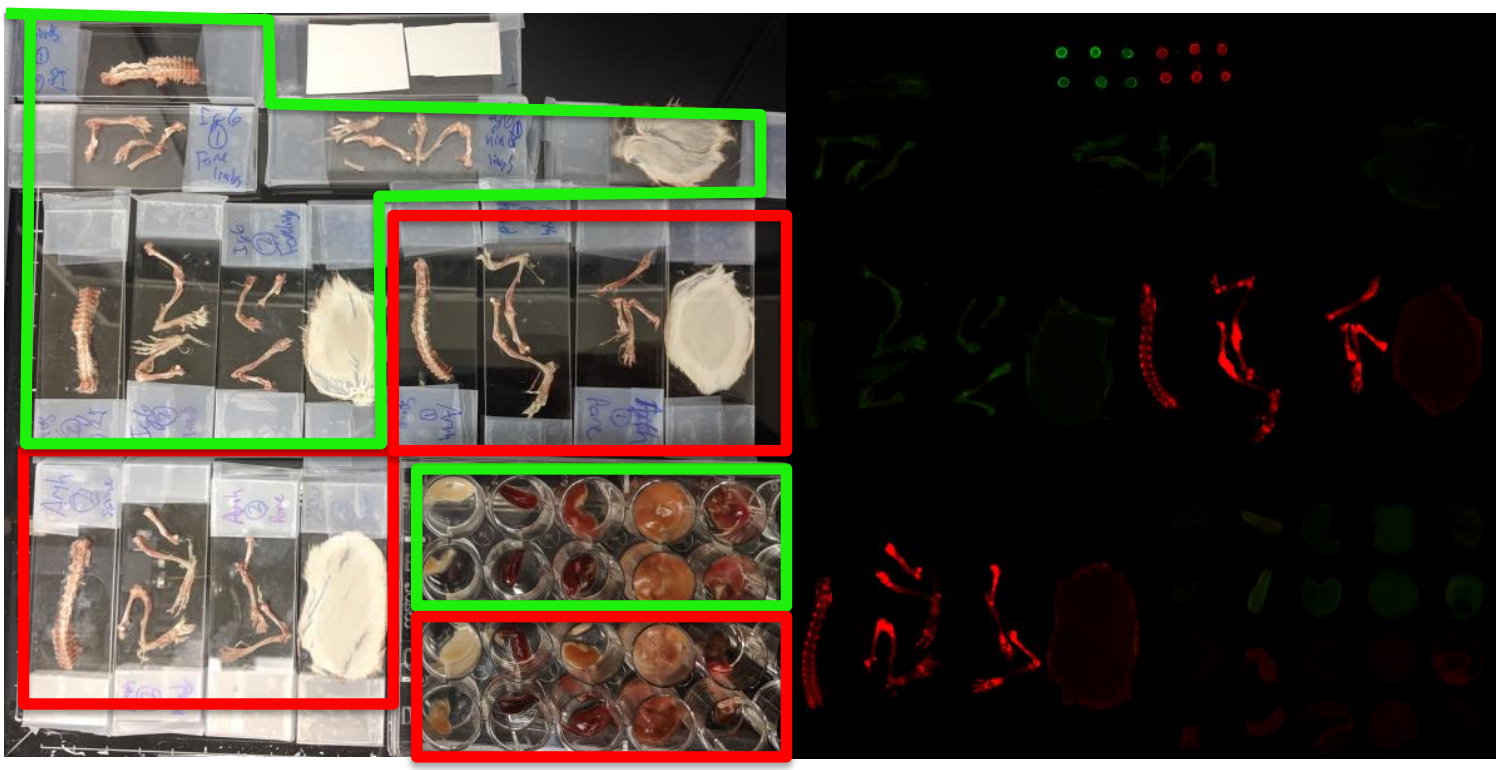

Arthrogen-680 mlgG-800

3 weeks

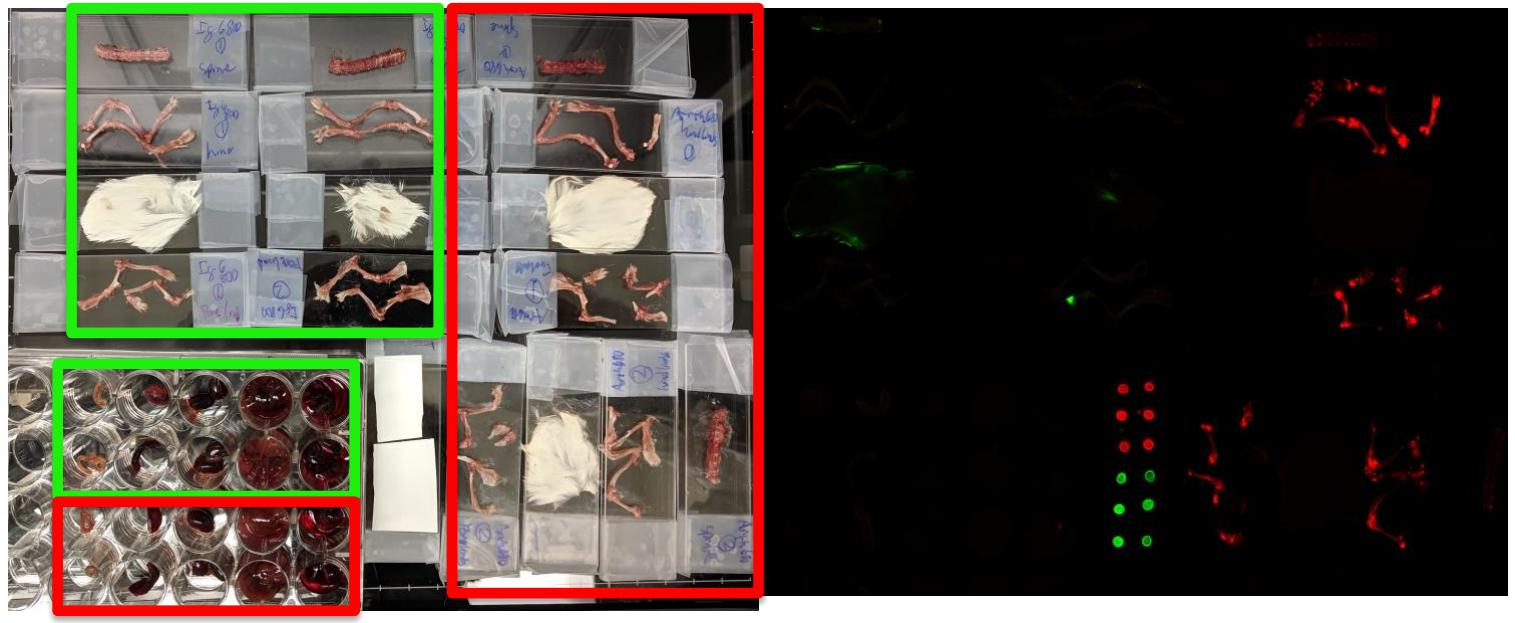

Arthrogen-680 mlgG-800

Fig. S2. Layout of the organs and the corresponding fluorescent scans (composite $700 \mathrm{~nm}$ and $800 \mathrm{~nm}$ ) for the experiment in Fig. 2D. Skin and bones are on slides and other organs are in a 24-well plate. Dots are the Arthrogen-680 and mouse IgG-800 dotted on the membrane at the same concentration. Organs (from left to right): intestine, spleen, kidneys, liver, lung/heart. 